\title{
TV/Series
}

9| 2016

Guerres en séries (I)

\section{Préface. Les séries télévisées américaines et la guerre contre la terreur}

\section{Marjolaine Boutet}

\section{(2) OpenEdition}

\section{Journals}

Édition électronique

URL : http://journals.openedition.org/tvseries/1327

DOI : 10.4000/tvseries.1327

ISSN : 2266-0909

\section{Éditeur}

GRIC - Groupe de recherche Identités et Cultures

Référence électronique

Marjolaine Boutet, «Préface. Les séries télévisées américaines et la guerre contre la terreur 》, TV/ Series [En ligne], 9| 2016, mis en ligne le 01 juin 2016, consulté le 22 septembre 2020. URL : http:// journals.openedition.org/tvseries/1327 ; DOI : https://doi.org/10.4000/tvseries.1327

Ce document a été généré automatiquement le 22 septembre 2020.

\section{(c) (i) (3) $\Theta$}

TV/Series est mis à disposition selon les termes de la licence Creative Commons Attribution - Pas d'Utilisation Commerciale - Pas de Modification 4.0 International. 


\title{
Préface. Les séries télévisées américaines et la guerre contre la terreur
}

\author{
Marjolaine Boutet
}

\section{Le 11 septembre, les médias et les séries télévisées}

1 Les attentats du 11 septembre 2001, et en particulier l'effondrement des tours du World Trade Center, retransmises en direct sur les écrans de télévision du monde entier, ont immédiatement interrogé le statut de l'événement et de l'image ${ }^{1}$. Paradoxalement, ce n'est pas tant le statut de l'image télévisée qui a d'abord été mise en cause, que celui de l'image et des récits cinématographiques. Dans The Terror Dream, Susan Faludi décrit cette impression persistante de « déjà-vu » face à ce qui était pourtant inédit :

We explained our failure to probe the same way that the Bush administration explained its failures to protect us: the attack was "unimaginable." Nothing like this had ever happened before, so we didn't know how to assimilate the experience. And yet, in the weeks and months to follow, we kept rummaging through the past to make sense of the disaster, as if the trauma of $9 / 11 \mathrm{had}$ stirred some distant memory, reminded us of something disturbingly familiar. As if we had been here before, after all $^{2}$.

2 Robert Altman, s'exprimant le 17 octobre 2001, rend le cinéma catastrophe responsable de la dimension spectaculaire des attentats, une affirmation souvent entendue dans les médias à l'époque :

The movies set the pattern, and these people have copied the movies. Nobody would have thought to commit an atrocity like that unless they'd seen it in a movie. How dare we continue to show this kind of mass destruction in movies. I just believe we created this atmosphere and taught them how to do it ${ }^{3}$. 
Ce discrédit du cinéma se prolonge par son incapacité à créer, dans les années qui suivent, de "grands films" à partir des événements : Oliver Stone avec World Trade Center (2006) et Paul Greengrass avec United 93 (2006) se rabattent ainsi sur des clichés hollywoodiens rassurants émotionnellement mais peu satisfaisants artistiquement et intellectuellement ${ }^{4}$. Susan Faludi se souvient :

In the electronic square, the gruesome visuals of the calamity replayed day after day, anniversary after anniversary, as if footage could substitute for fathoming, repetition for revelation. "Everything has changed" was our insta-bite mantra, recited in lieu of insight. Our media chattered on about "the death of irony" and "the death of postmodernism", without ever getting close to the birth of comprehension. The cacophony of chanted verities induced a kind of cultural hypnosis; Americans seemed to slip into a somnambulistic state. [...] By mid-2007, long after the nation had passed the five-year-anniversary mark of the attacks, we were still sleep-walking. Virtually no film, television drama, play, or novel on $9 / 11$ had begun to plumb what the trauma meant for our national psyche ${ }^{5}$.

Pour Claude Lanzmann, cette aporie cinématographique proviendrait de la qualité même de ces images "volées à la réalité6." Dans le même temps, la fascination esthétique et morbide pour ces images d'horreur dans un ciel bleu immaculé et au cœur d'une des plus belles villes du monde ne fait pas disparaitre le besoin de fiction, et le cinéma hollywoodien est indéniablement hanté par les événements, qu'il évoque au moyen de métaphores plus ou moins subtiles ${ }^{8}$. Le cinéma n'est évidemment pas le seul média à exprimer ce traumatisme collectif, qui n'a pas touché que la nation américaine et qui, par la suite, a frappé d'autres nations occidentales comme l'Espagne en 2004, la Grande-Bretagne en 2005, la France en 2015 et la Belgique en 2016.

Bien évidemment, les actes terroristes ne se réduisent pas à ces quelques événements, et ont un histoire qui remonte au moins à l'Antiquité, si on considère comme actes de terrorisme des actes de violence délibérés commis à l'encontre de civils à des fins politiques ${ }^{9}$. Néanmoins, les attentats du 11 septembre 2001 ont visé une pluralité de cibles, symboliques, économiques et psychologiques, caractéristiques de la stratégie terroriste contemporaine. Cette diversité de cibles frappées simultanément, le nombre de morts, l'ampleur des dégâts matériels et la résonance mondiale de l'événement ont permis à François Heisbourg de forger le concept d'hyperterrorisme ${ }^{10}$. Thierry Widemann explique :

Cet aspect de résonance est essentiel. La stratégie de terreur d'une organisation terroriste, dans la mesure où elle ne dispose que d'une capacité de destruction relativement limitée, exige que ses effets soient connus du plus grand nombre. D'où le rôle des médias, qui offrent au terrorisme la possibilité de réaliser une économie de moyens ${ }^{11}$.

6 Le traitement médiatique, amplifié quand les attentats frappent des nations développées où les journalistes sont très nombreux - voire lorsqu'eux-mêmes en sont les cibles comme à Paris le 7 janvier 2015, fait partie intégrante de la terreur produite, tandis que, paradoxalement, les images de « vraies guerres » se font plus rares et plus difficiles à obtenir. Dans une chronique sur France Culture, prononcée deux jours avant les attentats du 13 novembre 2015, autre journée d'attaques simultanées désignée par sa seule date, Xavier de la Porte dit que « toutes ces images [...] créent paradoxalement une nécessité de fiction», et une envie, entre autres, de "voir des séries qui, parce 
qu'elles ont pour elles le temps long, filment l'attente, l'ennui, l'irruption soudaine et parfois très ponctuelle, de la violence ([il] pense à Generation Kill par exemple) ${ }^{12}$. »

7 Il n'est pas anodin que le rédacteur en chef de rue89 cite une série télévisée comme fiction " réparatrice » face à l'horreur réelle, face à ces images inévitables diffusées et rediffusées sur les écrans multiples, des réseaux sociaux aux chaînes d'information en continu. Le 11 septembre survient en effet à un moment où le cinéma indépendant américain connaît d'importantes difficultés tandis que les séries télévisées sont en train de gagner une nouvelle légitimité artistique grâce à une complexité narrative grandissante, une audace esthétique de plus en plus assumée et l'engouement critique pour The Sopranos, diffusé sur la chaîne à péage HBO. La télévision, dans ses émissions d'information et de talk-shows ${ }^{13}$, a accompagné la population américaine dans son travail de deuil et les chaînes d'information en continu nourrissent depuis lors les moments de sidération décrits par Derrida à la suite de catastrophes de cette ampleur. Mais les séries télévisées, en tant que fictions longues qui s'inscrivent dans le rythme hebdomadaire des spectateurs, offrent davantage car elles permettent la sublimation, la transformation et l'appropriation du récit du traumatisme ${ }^{14}$. Grâce à leur aspect répétitif et familier, leur diffusion à intervalle fixe et le monde fictionnel à la fois complexe et relativement cohérent qu'elles proposent, les séries télévisées contemporaines se sont affirmées comme un vecteur culturel primordial de l'Occident en crise du XXIème siècle, et peut-être comme un lieu de gestion collective du traumatisme.

\section{La guerre contre la terreur dans les séries télévisées: détours, dispositifs et ambiguiités}

8 Si le premier article de ce numéro ${ }^{15}$ revient sur «Isaac et Ishmael ", l'épisode de The West Wing écrit par Aaron Sorkin au lendemain des attaques et diffusé trois semaines plus tard, et que le dernier article ${ }^{16}$ pointe les échos visuels des tours effondrées dans Homeland, ce qui nous intéresse ici est plus précisément la représentation de la réaction américaine à ce qui fut immédiatement interprété comme un acte de guerre : la guerre contre le terrorisme annoncée au soir même des attentats par le président George W. Bush, rebaptisée « guerre contre la terreur " dès le 20 septembre $2001^{17}$.

Or, Stephen Lacey et Derek Paget ont récemment souligné que cette guerre était bien plus une métaphore qu'une réalité, une façon de «mener des combats familiers sous de nouvelles bannières ${ }^{18}$ ", tandis que Stacy Takacs estime qu'elle était surtout destinée à rassurer la population américaine et à lui donner un échappatoire sur le plan affectif, sans fondement idéologique cohérent ${ }^{19}$. Dans le même ordre d'idée, la correspondante de guerre Megan Stack écrit :

The 'war on terror' never really existed. It was not a real thing [...] it was hollow, it was essentially a unifying myth for a complicated scramble of mixed impulses and social theories and night terrors and cruelty and business interests, all overhung with unassailable memory of falling skyscrapers $^{20}$.

Le sentiment d'irréalité, tant face à la surexposition des attentats qu'à la relative absence d'images des actions menées en Afghanistan et en Irak, est prégnant. Dès lors, pour reprendre l'interrogation de Vanessa Loubet-Poëtte, «comment, dans une 
actualité et un système médiatique abreuvés par tant d'images, trouver d'autres moyens de représentation d'une guerre si spécifique que celle menée contre le terrorisme, sans tomber dans la surenchère ou l'altération ${ }^{21}$ ?» La réponse apportée par les séries télévisées étudiées dans la première partie de ce numéro (The West Wing, The Newsroom, Generation Kill et In Treatment) semble être celle du détour, voire du détournement, visuel ou narratif. C'est ainsi que la référence à Hiroshima mon amour d'Alain Resnais (1959) se retrouve dans de nombreux articles pour interroger ce qui a été vu ou pas, compris ou pas, de la catastrophe et de ses conséquences.

La linguiste Vanessa Loubet-Poëtte analyse ainsi le traitement que le scénariste démiurge Aaron Sorkin a réservé au 11 septembre 2001 puis à l'assassinat d'Oussama Ben Laden le 2 mai $2011^{22}$ dans deux de ses séries : The West Wing (NBC, 1999-2006) avec le célèbre épisode spécial intitulé « Isaac and Ishmael » diffusé le 3 octobre 2001, et The Newsroom (HBO, 2012-2014) avec l'épisode « 5/1» diffusé le 5 août 2012. Elle montre notamment comment, grâce à la multiplication des dialogues, à la véridiction, à la dramaturgie et aux effets rhétoriques, les deux épisodes ont un effet cathartique qui affirme la victoire de l'intelligence sur la barbarie et s'inscrivent dans l'œuvre singulière de leur auteur.

13 Les américanistes Sébastien Lefait et Monica Michlin proposent ensuite deux interprétations complémentaires de la mini-série Generation Kill (HBO, 2008) de David Simon, adaptée du livre écrit en 2004 par le journaliste embarqué Evan Wright, à la suite de son expérience de l'invasion de l'Irak de 2003 aux côtés d'un bataillon de Marines. Sébastien Lefait montre comment la série met en scène les multiples dispositifs optiques et techniques qui tiennent les soldats à distance de la guerre qu'ils mènent, questionnant par là même notre propre rapport aux images, à la perception et/ou l'aperception. Monica Michlin explore ensuite les ambiguïtés délibérées de cette mise en scène et de ces dispositifs, entre esthétisation de la virilité et de la violence et dénonciation de la cruauté et de l'ennui, pour finir, comme dans The Wire (HBO, 2002-2008) sur son démantèlement réflexif.

14 Enfin, Sarah Hatchuel, spécialiste de littérature et de cinéma anglophones, analyse comment le dispositif particulier de la série In Treatment (HBO, 2008-2010), adaptation américaine de la série israélienne BeTipul (HOT3, 2005-2008), est mis en danger par la guerre contre la terreur comme trauma. En effet, la série met en scène une séance de psychanalyse quotidienne en suivant quatre patients différents qui reviennent le même jour chaque semaine (le vendredi, c'est au tour de Paul, le psychanalyste, de faire son analyse face à une collègue). La singularité et la complexité du dispositif narratif contrastent avec la simplicité de la mise en scène (toutes les séances sont à huis clos, à l'intérieur du cabinet du psychanalyste, avec de très rares interventions extérieures et très peu d'échappées visuelles réelles ou fantasmées). Ce dispositif est perturbé dans la première saison par un patient peu ordinaire, celui du mardi : un pilote de retour d'Irak en plein déni de son traumatisme. La conclusion abrupte reflète alors à la fois la puissance et l'impuissance du récit pour soigner le traumatisme, à l'intérieur comme à l'extérieur de la diégèse.

15 La deuxième partie de ce volume est consacrée à des analyses originales de deux séries profondément associées à la guerre contre la terreur : 24 heures chrono (Fox, 2001-2010 ; 2014) et Homeland (Showtime, depuis 2011). Monica Michlin et Ophir Lévy, philosophe et historien du cinéma, réfléchissent tous deux au sens et aux effets du split-screen dans 
24 heures chrono. Tous deux y voient un dispositif destiné à manipuler le spectateur, symbolisant la fracture paranoïaque de l'écran, du héros et par-delà, de l'Amérique elle-même face à la guerre et à la torture pour Monica Michlin, instrument d'une forme de sadisme narratif qui accroît à la fois la confusion et le plaisir des téléspectateurs pour Ophir Lévy. L'américaniste Alexis Pichard et moi-même, historienne de formation, nous intéressons ensuite au tournant narratif de la série dans sa septième saison, diffusée au début de l'année 2009. Cette première saison post-grève des scénaristes et post-Bush incarne pour Alexis Pichard les hésitations et les ambiguïtés de l'Amérique d'Obama, en particulier dans sa posture ambivalente face à l'usage de la torture par le héros de la série. Je m'intéresse pour ma part aux personnages féminins de cette septième saison qui, tout en détournant les stéréotypes traditionnels, amorcent un tournant narratif qui aurait pu aboutir au remplacement de Jack Bauer par un alter ego féminin dans la lutte contre le terrorisme, mais n'a sans doute pas été mené au bout de sa logique pour des raisons économiques.

Il faut attendre Homeland, la production suivante d'Howard Gordon et Alex Gansa (ce dernier ayant d'ailleurs rejoint 24 heures chrono pour sa septième saison) pour voir une héroïne féminine au cœur de la lutte anti-terroriste : Carrie Mathison (Claire Danes). Pauline Blistène et Olivier Chopin, spécialistes de relations internationales, montrent que la première saison de la série peut être lue à deux niveaux : au premier degré comme une dramatisation de la guerre contre la terreur et des nouvelles menaces sur le sol américain, mais aussi, au second degré comme une allégorie de la crise identitaire que connait la démocratie américaine depuis le 11 septembre, et des mutations de son sentiment national. Puis Alexis Pichard nous explique en quoi les quatre premières saisons d'Homeland permettent aux téléspectateurs de digérer le choc du 11 septembre, puis de réfléchir à la part de culpabilité américaine dans les attaques dont le pays est victime, pour finalement interroger, non sans ambiguïté, les fondements de l'islamophobie.

Il est révélateur que la plupart des articles du présent numéro portent sur des épisodes diffusés à partir de 2008, comme si la fin du second mandat de George W. Bush permettait la distance nécessaire pour la mise en fiction de la guerre contre la terreur. Toutefois, cette politique a été prolongée par son successeur démocrate, avec finalement plus de continuités que de ruptures. Il parait évident qu'au moment où la présidence Obama s'achève, ces fictions ne sont qu'une première digestion d'une évolution de la nation américaine dont seul le temps permettra de prendre toute la mesure. A suivre, donc, comme les épisodes de nos séries préférées.

Ce numéro est composé des versions écrites et étendues d'une partie des communications prononcées lors de la journée d'études du cycle Philoséries consacrée à 24 heures chrono en 2010, organisée à l'Ecole Normale Supérieure de la rue d'Ulm par Sylvie Allouche et Sandra Laugier, et lors du colloque «Guerres en séries » organisé en juin 2014 à Amiens par Marjolaine Boutet avec le soutien du Centre d'Histoire des Sociétés, des Sciences et des Conflits de l'Université de Picardie-Jules Verne, de GUEST-Normandie (Groupe Universitaire d'Etudes sur les Séries Télévisées basé en Normandie), de la région Picardie et d'Amiens métropole. 


\section{BIBLIOGRAPHIE}

BAUDRILLARD, Jean, L'Esprit du terrorisme, Paris, Editions Galilée, 2002.

BORRADORI, Giovanna, DERRIDA, Jacques et HABERMAS, Jürgen, Le « concept » du 11 septembre. Dialogues à New York (octobre-décembre 2001) avec Giovanna Borradori. Paris, Galilée, « La philosophie en effet ", 2003.

BRENDER Olivia, «Fiction et événement : le 11 septembre dans les séries télévisées américaines, 2001-2003 », Bulletin de l'Institut Pierre Renouvin, n² 26, février 2007, p. 81-96. URL :

www.cairn.info/revue-bulletin-de-l-institut-pierre-renouvin1-2007-2-page-81.htm. DOI : 10.3917/bipr.026.0081. Consulté le 30 mai 2016.

ELSAESSER Thomas, «Reports and debate. Postmodernism as mourning work », Screen, $\mathrm{n}^{\circ} 42.2$, 2001, p. 193-201.

FALUDI, Susan, The Terror Dream: Fear and Fantasy in Post 9/11 America, Londres, Atlantic Books, 2008. GERVAIS, Bertrand, « Déjouer le spectacle de la violence. Représenter les événements du 11 septembre 2001 », E-rea, 9.1, 2011, URL : http://erea.revues.org/1944 ; DOI : 10.4000/erea.1944 consulté le 25 mai 2016 GRUSIN Richard, Premediation: Affect and Mediality After 9/11, New York, Palgrave Macmillan, 2010. HAMMOND, Philip, Screens of Terror: Representations of War and Terrorism in Film and Television Since 9/11, Bury St Edmunds, Arima Publishing, 2011.

HENNINGER, Laurent, WIDEMANN, Thierry, Comprendre la guerre : histoire et notions, Paris, Perrin, 2012 .

LACEY Stephen, PAGET, Derek (dir.), The War on Terror: post-9/11 television drama, docudrama and documentary, Cardiff, University of Wales Press, 2015.

LAMY Aurélia, « Les spécificités du traitement médiatique dans l'urgence. L'exemple des attentats du 11 septembre $2001 »$, Communication et organisation, $n^{\circ} 29,2006$, p. 108-122.

SOULADIÉ, Vincent, « Adapter le 11 septembre 2001 au cinéma : Limites et écarts de la représentation dans World Trade Center (Oliver Stone, 2006) », Miranda [Online], 8 | 2013, URL : http://miranda.revues.org/3556 (consulté le 25 mai 2016)

STEINER, Tobias, « Dealing with a Nation's Trauma: Allegories on 9/11 in Contemporary Serial US Television Drama Narratives and the case of Homeland ", Mémoire de Master dirigé par Janet McCabe, Birbeck College, University of London, 2012. Disponible sur https://www.academia.edu/ 2272123/

Dealing_with_a_Nations_Trauma_Allegories_on_9_11_in_Contemporary_Serial_US_Television_Drama_Narratives_and_the_case_of_H Consulté le 31 mai 2016.

TAKACS, Stacy, Terrorism TV : Popular Entertainment in Post-9/11 America, Lawrence, University of Kansas Press, 2012. 


\section{NOTES}

1. Cf. notamment Jean Baudrillard, L'Esprit du terrorisme, Paris, Editions Galilée, 2002 et Giovanna Borradori, Jacques Derrida et Jürgen Habermas, Le « concept » du 11 septembre. Dialogues à New York (octobre-décembre 2001) avec Giovanna Borradori. Paris, Galilée, « La philosophie en effet », 2003.

2. Susan Faludi, The Terror Dream, Londres, Atlantic Books, 2008 [2007], p. 3. Voir aussi Richard Grusin, Premediation: Afect and Mediality After 9/11, New York, Palgrave Macmillan, 2010.

3. Robert Altman, Associated Press Wire Story, 17 octobre 2001, cité in Stephen Prince, Classical Film Violence Designing And Regulating Brutality In Hollywood Cinema 1930-1968, Londres, Rutgers University Press, 2003, p. 286.

4. Cf. Vincent Souladié, "Adapter le 11 septembre 2001 au cinéma : Limites et écarts de la représentation dans World Trade Center (Oliver Stone, 2006) », Miranda, n8, 2013, URL : http:// miranda.revues.org/3556 consulté le 25 mai 2016.

5. Susan Faludi, The Terror Dream, Londres, Atlantic Books, 2008 [2007], p. 2.

6. Patrice Blouin, Franck Nouchi, Charles Tesson, «Claude Lanzmann : Sur le courage », Cahiers du cinéma, $\mathrm{n}^{\circ}$ 561, octobre 2001, p. 56.

7. Jean Baudrillard, op. cit., p. 39.

8. Cf. Tom Pollard, Hollywood 9/11: Superheroes, supervillains, and super disasters Boulder (Colorado), Paradigm Publishers, 2011 ; Douglas Kellner, Cinema wars: Hollywood film and politics in the BushCheney era Londres, Wiley-Blackwell, 2010 ; Helena Vanhala, The depiction of terrorists in blockbuster Hollywood films, 1980-2001: An analytical study Jefferson, McFarland, 2010 ; Robert Cettl, Terrorism in American cinema: An analytical filmography, 1960-2008 Jefferson, McFarland, 2009 ; Stephen Prince, Firestorm: American film in the age of terrorism, New York, Columbia University Press, 2009, Wheeler W. Dixon, Film and television after 9/11, Carbondale, Southern Illinois University Press, 2004, Stieg A. Nohrstedt et al., U.S. and the others: Global media images on "The War on Terror", Göteborg, Nordicom, 2004.

9. Thierry Widemann, " Le terrorisme : un phénomènepolymorphe ", in Comprendre la guerre: histoire et notions, Laurent Henninger et Thierry Widemann, Paris, Perrin, 2012, p. 145-147.

10. François Heibsourg, Hyperterrorisme : la nouvelle guerre, Paris, Odile Jacob, 2001.

11. Thierry Widemann, "Le terrorisme : un phénomènepolymorphe ", in Comprendre la guerre: histoire et notions, Laurent Henninger et Thierry Widemann, Paris, Perrin, 2012, p. 149.

12. Xavier de la Porte, "La guerre comme vous l'avez trop vue », France Culture, 11 novembre 2015. http://www.franceculture.fr/emissions/le-monde-selon-xavier-de-la-porte/la-guerrecomme-vous-l-avez-deja-trop-vue (consulté le 5 mai 2016)

13. Aurélia Lamy, «Les spécificités du traitement médiatique dans l'urgence. L'exemple des attentats du 11 septembre 2001 ", Communication et organisation, n²9, 2006, p. 108-122; Thomas Elsaesser, "Reports and debate. Postmodernism as mourning work», Screen, $\mathrm{n}^{\circ} 42.2,2001$, p. 193-201.

14. Cf. Olivia Brender, "Fiction et événement : le 11 septembre dans les séries télévisées américaines, 2001-2003 », Bulletin de l'Institut Pierre Renouvin 2/2007 ( ${ }^{\circ}$ 26) , p. 81-96 https:// www.cairn.info/revue-bulletin-de-l-institut-pierre-renouvin1-2007-2-page-81.htm

15. Vanessa Loubet-Poëtte, "Conflits, filtres et stratégies d'évitement : la représentation du 11 septembre et de ses conséquences dans deux séries d'Aaron Sorkin, The West Wing (NBC, 1999-2006) et The Newsroom (HBO, 2012-2014) », TV/Series, ${ }^{\circ} 9$, juin 2016. http:// tvseries.revues.org/1217

16. Alexis Pichard, « Homeland : un antidote à la guerre contre le terrorisme ? ", TV/Series, $n^{\circ} 9$, juin 2016. http://tvseries.revues.org/1284

17. George W. Bush, «Address to the Nation on the September 11 Attacks » et «Address to the Joint Session of the 107th Congress", Selected Speeches of President George W. Bush, 2001-2008, Archives nationales américaines disponibles à l'adresse url: https://georgewbush- 
whitehouse.archives.gov/infocus/bushrecord/documents/

Selected_Speeches_George_W_Bush.pdf consultée le 31 mai 2016.

18. Stephen Lacey et Derek Paget, The War on Terror: post 9/11 television drama, docudrama and documentary, Cardiff, University of Wales Press, 2015, p. iv-v.

19. Stacy Takacs, Terrorism TV : Popular Entertainment in Post-9/11 America, Lawrence, University of Kansas Press, 2012, p. 26.

20. Megan Stack, Every Man in This Village is a Liar: An Education in War, Londres, Bloomsbury, 2012, p. 15.

21. Vanessa Loubet-Poëtte, op.cit., §4.

22. L'assassinat ayant eu lieu vers 1 heure du matin le 2 mai, les Etats-Unis ont retenu la date du $1^{\mathrm{er}}$ mai qui correspond à leur heure locale, d'où le titre de l'épisode choisi par Sorkin.

\section{INDEX}

Mots-clés : guerre contre la terreur, histoire, 11 septembre, violence, terrorisme, médias, cinéma, Obama Barack, Bush George W., traumatisme

Keywords : War on Terror, History, violence, 9/11, terrorism, media, cinema, Obama Barack, Bush George W., trauma

\section{AUTEUR}

\section{MARJOLAINE BOUTET}

Marjolaine Boutet est maître de conférences en Histoire contemporaine à l'université de Picardie-Jules Verne. Spécialiste de l'histoire des séries télévisées, elle est l'auteure de Les Séries Télé pour les Nuls (First, 2009) et Cold Case : la mélodie du passé (PUF, 2013) ainsi que co-auteure de Seriescopie : guide thématique des séries télévisées (Ellipses, 2011) avec Pierre Sérisier et Joël Bassaget. Elle s'intéresse particulièrement à la représentation de l'Histoire et des conflits dans les séries télévisées, qu'ils soient militaires, politiques ou symboliques.

Marjolaine Boutet is Associate Professor in Contemporary History at the University of PicardieJules Verne. She specializes in TV series and is the author of Les Séries Télé pour les Nuls (First, 2009) and Cold Case : la mélodie du passé (PUF, 2013), as well as a co-author of Seriescopie : guide thématique des séries télévisées (Ellipses, 2011) with Pierre Sérisier and Joël Bassaget. Her research focuses on the representation of History and conflicts on screen. 